\title{
O fabrico das poliolefinas em Portugal
}

\section{Introdução}

$\mathrm{O}$ mundo à nossa volta sempre conteve polímeros. Contudo antes da Segunda Guerra Mundial a maior parte dos produtos poliméricos ocorriam na natureza. Actualmente coexistem as duas fontes: a natural e a artificial.

Os plásticos, compostos de alto peso molecular, podem ser classificados em termoplásticos e termo-endurecíveis. Os primeiros, são produzidos na EPSI. Quando sujeitos ao calor fundem e podem ser moldados, quando reaquecidos, podem ser novamente moldados. Os outros sofrem transformaçð̃es químicas que não permitem essa operação dupla.

Em 1933 Gibson e Fawcett começaram a estudar os processos de polimerização do etileno a alta pressão nos laboratórios da ICI em Inglaterra. A produção industrial começou em 1942. Posteriormente, em 1953, nova técnica a baixa pressão foi desenvolvida por Ziegler, seguindo-se a da Philips e Satandard Oil. A produção industrial deste outro tipo de polietileno, chamado linear começou em 1956. Em 1954 o prof. Natta descobriu que o catalisador desenvolvido por Ziegler, tinha um efeito estereoespecífico durante a polimerização do polipropileno formando-se uma elevada percentagem de polipropileno cristalino-isotático. Com base nessa descoberta, começou a sua produção industrial em 1959.

O polietileno continua a ser o polimero de maior consumo no mundo. Actualmente ele é disponível numa grande variedade de formas estruturais, como homopolímero, copolímero e em misturas de polimeros. Pode-se dizer que as suas propriedades podem ser ajustadas à medida desejada.

O polipropileno, menor em produção que o $\mathrm{PE}$, está tendo grande sucesso em rápido crescimento.

\section{Produção e consumo de polietileno + polipropileno} PRODUÇÃO MUNDIAL

\begin{tabular}{|c|c|c|c|c|c|}
\hline & 1950 & 1960 & 1976 & 1980 & 1984 \\
\hline $\begin{array}{l}\text { EUA } \\
\text { Europa Ocidental } \\
\text { Japão } \\
\text { Mundo }\end{array}$ & $\begin{array}{r}25 \\
5 \\
- \\
-\end{array}$ & $\begin{array}{c}364 \\
260 \\
120 \\
-\end{array}$ & $\begin{array}{rl}5 & 204 \\
6 & 135 \\
1 & 600 \\
14 & 420^{*}\end{array}$ & $\begin{array}{r}6950 \\
7413 \\
2787 \\
19876\end{array}$ & $\begin{array}{l}8280 \\
8641 \\
3237 \\
-\end{array}$ \\
\hline
\end{tabular}

- Excepto paises do Leste Europeu.

PRODUÇÃO, CAPACIDADE E CONSUMO NA EUROPA OCIDENTAL

\begin{tabular}{|c|c|c|c|c|c|c|}
\hline \multicolumn{7}{|c|}{$\times 10^{3}$ Ton } \\
\hline & 1976 & 1980 & 1982 & 1983 & 1984 & 1985 \\
\hline $\begin{array}{l}\text { Produção } \\
\text { Capacidade } \\
\text { Consumo }\end{array}$ & $\begin{array}{ll}6 & 135 \\
8 & 585 \\
5 & 130\end{array}$ & $\begin{array}{r}7413 \\
10999 \\
6542\end{array}$ & $\begin{array}{r}7090 \\
10486 \\
6513\end{array}$ & $\begin{array}{r}7747 \\
10894 \\
6955\end{array}$ & $\begin{array}{l}8641 \\
7 \overline{159}\end{array}$ & $\begin{array}{c}10 \overline{7} 68 \\
-\end{array}$ \\
\hline
\end{tabular}

PRODUÇÃO E CONSUMO NALGUNS PAÍSES DA EUROPA OCIDENTAL

\begin{tabular}{|c|c|c|c|c|c|c|}
\hline \multicolumn{7}{|c|}{ x $10^{3}$ Ton } \\
\hline . & \multicolumn{2}{|c|}{1978} & \multicolumn{2}{|c|}{1980} & \multicolumn{2}{|c|}{1982} \\
\hline & Produção & Consumo & Produção & Consumo & Produção & Consumo \\
\hline $\begin{array}{l}\text { Alemanha Ocidental } \\
\text { França } \\
\text { Itália } \\
\text { Benelux } \\
\text { Espanha } \\
\text { Portugal }\end{array}$ & $\begin{array}{rr}1715 \\
1100 \\
1030 \\
1095 \\
410 \\
& 0\end{array}$ & $\begin{array}{r}1363 \\
860 \\
930 \\
450 \\
460 \\
120\end{array}$ & $\begin{array}{r}1615 \\
1285 \\
990 \\
1625 \\
543 \\
0\end{array}$ & $\begin{array}{r}1485 \\
955 \\
1170 \\
565 \\
450 \\
110\end{array}$ & $\begin{array}{r}1378 \\
1205 \\
875 \\
1815 \\
537 \\
65\end{array}$ & $\begin{array}{r}1445 \\
905 \\
1078 \\
534 \\
441 \\
123\end{array}$ \\
\hline
\end{tabular}


CAPACIDADE DE PRODUÇÃo POR TIPOS DAS MAIORES COMPANHIAS EUROPEIAS NO ANO DE 1985

\begin{tabular}{|c|c|c|c|c|c|}
\hline \multicolumn{6}{|c|}{$x 10^{3}$ Ton } \\
\hline \multicolumn{2}{|c|}{ PEBD + LLDPE } & \multicolumn{2}{|c|}{ PEAD } & \multicolumn{2}{|c|}{ PP } \\
\hline $\begin{array}{l}\text { Enichemie } \\
\text { Dow } \\
\text { Empetrol } \\
\text { Essochemie }\end{array}$ & $\begin{array}{l}690 \\
563 \\
638 \\
480\end{array}$ & $\begin{array}{l}\text { Hoechst } \\
\text { Solvay } \\
\text { Enichemie } \\
\text { Petrochem }\end{array}$ & $\begin{array}{l}425 \\
225 \\
210 \\
230\end{array}$ & $\begin{array}{l}\text { Himont } \\
\text { Hoechst } \\
\text { ICI } \\
\text { Shell }\end{array}$ & $\begin{array}{l}565 \\
310 \\
290 \\
375\end{array}$ \\
\hline
\end{tabular}

CAPACIDADE dE PROdUÇÃo MUNDIAL DE PE + PP

$\mathrm{x} 10^{3}$ Ton

\begin{tabular}{c|c|c|c|c}
\hline 1980 & 1982 & 1983 & 1984 & $\begin{array}{c}1988 \\
\text { (previsão) }\end{array}$ \\
\hline 27420 & 31145 & 29666 & 34597 & 37924 \\
\hline
\end{tabular}

\section{Processos de fabrico usados na EPSI, em Portugal}

PEBD (polietileno de baixa densidade, ramificado)

O processo usado no PEBD da EPSI é o "ethylene plastique'". Trata-se dum processo de fabricação de polietileno ramificado obtido por polimerização do etileno com $\mathrm{P} \simeq 2000$ bares e $\mathrm{T} \simeq 250^{\circ} \mathrm{C}$.

O etileno com grau de pureza $>99.9 \%$ é comprimido em duas etapas antes de entrar no reactor. Neste, são introduzidos também os iniciadores da reacção (peróxidos orgânicos) e, para certas qualidades, outros comonómeros e agentes de transferência. À saida do reactor, a mistura de polietileno separa-se do etileno não polimerizado, num separador em duas fases, uma gasosa contendo etileno e polímero de baixo peso molecular, que é reciclado e uma fase líquida contendo polietileno em etileno dissolvido que se deposita no fundo do separador. O etileno após arrefecimento é recomprimido a elevada pressão e reenviado ao reactor. O polietileno depositado no fundo do separador alimenta uma extrusora que serve para granular o polimero misturando-o com aditivos cujas composição e teor variam consoante as aplicações. O polímero depois de sair da extrusora é seco, classificado e transportado pneumaticamente para silos de controle, antes da homogeneização. Destes, após análise de controle de qualidade, passa à ensacagem onde é metido em sacos de $25 \mathrm{~kg}$ em lotes de cerca de 200 ton.

\section{PEAD (polietileno de alta densidade)}

$\mathrm{O}$ processo usado na EPSI é o processo " $\mathrm{CX}$ da MPC" (Mitsui Petrochemical Industries). É um tipo "Ziegler" usando catalisador de alta actividade (2. ${ }^{\text {a }}$ geração), em suspensão de hexano, a baixa pressão. Usa um ou dois reactores, em série ou paralelo, conforme os tipos pretendidos.

No reactor entra o etileno com grau de pureza $99.9 \%$ e outros comonómeros, o catalisador e o hidrogénio. Controlando a pressão $\left(2-10 \mathrm{~kg} / \mathrm{cm}^{2}\right)$, a temperatura a cerca de $80^{\circ} \mathrm{C}$ e a concentração dos catalisadores, obtém-se uma suspensão de polímero em hexano. O gás de reciclagem (etileno não reagido), previamente separado do hexano e do polímero é introduzido novamente no reactor. A pasta de polímero formada é centrifugada, separando-se do hexano contendo baixo polímero dissolvido. Depois de seco, em secador rotativo em contracorrente de azoto quente, o pó é aditivado para estabilização passando da tremonha para a extrusora, onde é fundido e granulado, depois do corte em água. Os granulados depois de secos são homogeneizados, procedendo-se ao controle de qualidade e poste- rior ensacamento em sacos de $25 \mathrm{~kg}$ e lotes de cerca de 200 ton.

\section{PP (polipropileno)}

O processo de fabrico usado na EPSI é um processo em suspensão, de Ziegler/Natta, modificado, usando catalisador de fraca actividade (1. a geração). É um processo com custos mais elevados, com vários reactores em série e diversas etapas de polimerização, desactivação do catalisador, lavagem e purificação do polimero.

O propileno no estado líquido, e por vezes o comonómero etileno, o heptano, o catalisador e hidrogénio alimentam continuamente os reactores. As condiçðes de polimerização variam ao longo dos reactores e em conformidade com os graus pretendidos com pressão 1 a 10 bares e temperatura cerca de $70^{\circ} \mathrm{C}$. No final há que desactivar os restos do catalisador com metanol e por elevação da temperatura. A suspensão do polímero passa depois a uma coluna de lavagem para remoção dos restos do catalisador. Segue-se o tanque de extracção, onde lhe é retirado, por dissolução em heptano aquecido, o polipropileno atático. $\mathrm{O}$ produto de primeira qualidade, polipropileno isotático, após centrifugação para separação do heptano com atático dissolvido, vai para os secadores onde é seco por contracorrente de azoto quente. O pó de PP é misturado com aditivos passando para a tremonha e, desta, para a extrusora para ser fundido e granulado, após homogeneização e controle de qualidade é ensacado em sacos de $25 \mathrm{~kg}$ e lotes de cerca de 60 ton.

\section{Custos de fabrico em percentagem relativa $(\%)$}

\begin{tabular}{lccc}
\hline & PEBD & PEAD & PP \\
\hline Matérias-primas & 77 & 74,5 & 64 \\
Energia + Utilidades & 10,5 & 12 & 18 \\
Mão-de-obra & 10 & 8.5 & 12 \\
Diversos* & 2.5 & 5 & 6 \\
\hline
\end{tabular}

- Aditivos + solventes + catalisadores.

4. Características básicas e sua influência nas propriedades gerais

PEBD

- Índice de fluidez (MI) que está relacionado com o peso molecular médio influencia a processabilidade e propriedades mecânicas.

- Densidade (d) que está relacionada com a cristalinidade.

As características gerais desta resina são:

- baixa densidade. 
- boa processabilidade.

- alta resistência ao impacto.

- excelente resistência química aos ácidos e bases.

- macio e flexível mesmo a baixas temperaturas.

- boas propriedades dieléctricas.

$P E A D$

Indice de fluidez (MI). Quando este diminui, aumenta o peso molecular médio e com ele o alongamento e as propriedades mecânicas: resistência ao impacto, ao rasgamento, a fissuração. Por outro lado, diminui a processabilidade.

Densidade (d). Esta aumenta com a cristalinidade que, por sua vez, reflecte certas propriedades importantes neste polimero:

- Maior rigidez e dureza.

- Pior resistência ao choque e ao aparecimento de fendas (fissuração).

As características gerais desta resina são:

- elevada cristalinidade com densidade entre 0.94 e 0.97 .

- boa processabilidade (melhor que a do PP).

- boa resistência a baixas temperaturas.

- maior rigidez e melhores propriedades mecânicas que o PEBD.
- pouco transparente.

PP

Existem dois tipos de polipropilenos - homopolímero e copolímero (bloco e estatístico - caso da EPSI). As características básicas são o índice de fluidez (MI) que dá uma ideia do peso molecular médio e o II (indice de isotaticidade) que é a percentagem de isotático. Com estas duas características se avalia a extrudabilidade e injectabilidade da resina.

Características gerais:

- as melhores propriedades mecânicas das três poliolefinas.

- boa resistência ao calor, podendo sofrer esterilização.

- excelente resistência mecânica na estabilidade dimensional dos objectos acabados.

- boa resistência química especialmente aos ácidos.

- muito transparente quando usado em filme.

- elevada resistência a fissuração.

- boa resistência a deformação sob carga.

5. Características globais das três poliolefinas (Ver quadro anexo)

\section{Vantagens e desvantagens comparativas do PEBD, PEAD e PP}

\begin{tabular}{|c|c|c|}
\hline Polimero & Vantagens & Desvantagens \\
\hline PEBD & $\begin{array}{l}\text { - Boa Processabilidade } \\
\text { - Boa resistência ao choque } \\
\text { - Inércia química } \\
\text { - Leve }\end{array}$ & $\begin{array}{l}\text { - Sensibilidade à fissuração em meio tenso activo } \\
\text { - Fraca resistência mecânica } \\
\text { - Permeabilidade aos gases }\end{array}$ \\
\hline PEAD & $\begin{array}{l}\text { - Maior rigidez que a do PEBD, mas inferior ao PP } \\
\text { - Pouco permeável à água } \\
\text { - Boa resistência ao frio e ao calor } \\
\text { - Boa resistência às radiaçðes } \\
\text { - Mais resistente à fissuração que o PEBD }\end{array}$ & - Propriedades mecânicas entre as do PEBD e PP \\
\hline PP & $\begin{array}{l}\text { - Muito leve } \\
\text { - Boa resistência mecânica (tensão ruptura e abrasão) } \\
\text { - Excelente resistência à flexão } \\
\text { - Boa resistência a temperaturas elevadas } \\
\text { - Boa resistência química e ausência de fissuração } \\
\text { - Bom aspecto. Dificilmente riscável }\end{array}$ & $\begin{array}{l}\text { - Fragilidade a baixas temperaturas } \\
\text { - Facilmente degradável, sem aditivação }\end{array}$ \\
\hline
\end{tabular}

\section{Aplicações}

\section{$P E B D$}

Embalagem sob forma de manga ou filme:

Sacos para produtos alimentares. Sacos para compras

"shopping bag"' (roupa, calçado, etc.), sacos de grande capacidade (adubo, farinha, sal, etc.).

Filme retráctil para enfardamento ou paletização. Filme para construção civil. Filme para estufas agrícolas e "paillages".

Cápsulas de rolhagem.

Produtos domésticos (pás, caixotes do lixo, etc.).

Tubos de creme leve.

Tubos de rega ou irrigação.

Revestimento de cabos telefónicos e eléctricos.

\section{PEAD}

Vasilhame para garrafas e caixas de manutenção.

Monofilamento e ráfias para cordas, redes e sacaria.

Frascos de detergentes, produtos de limpeza e cosméticos.

Grandes tambores para 150 e 200 litros.

Artigos domésticos em geral, brinquedos, peças eléctricas e industriais.

Filmes finos para embalagens alimentares.

Tubos para água ou esgotos de grande diâmetro, para regiðes frias.
Garrafas de leite esterilizado.

$P P$

Filme para Ráfia e monofilamentos em sacaria média e grandes dimensð̄es.

Filme para base de carpetes.

Peças técnicas e industrais para trabalharem a temperaturas elevadas (máquinas de lavar, secadores de cabelo, aspiradores, etc.).

Peças para automóveis e carcaças de bateria (copolímero).

Tubos para água quente.

Acessórios para casas de banho.

Aparelhos cirúrgicos necessitando de esterilização.

Corpos ocos para sumos de fruta, óleos.

\section{BIBLIOGRAFIA}

PCX Training Test-Book, MPC.

Modern Plastics/International

Chem Systems International, PPE C. Thermoplastics.

Principles of Polymer Systems

Experiments in Polymer Science, Fred W. Billmeyer, J.R.

Modern Plastics Encyclopedia, 83/84.

Plastics the Next Ten Years Interplas, London 1978.

ECN/European Chemical News. 


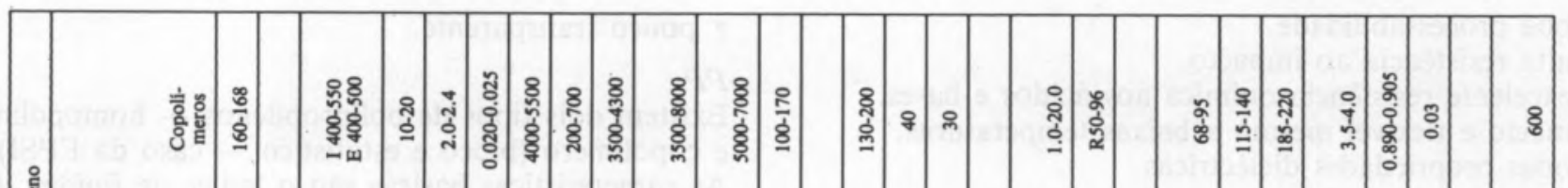

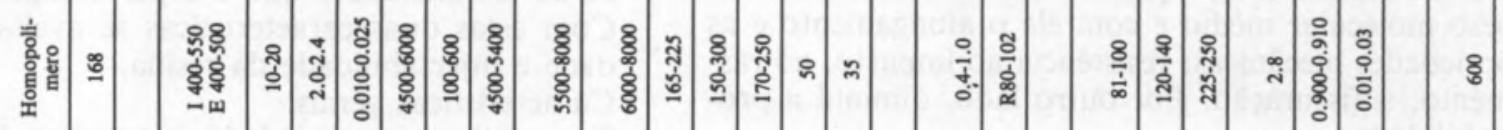

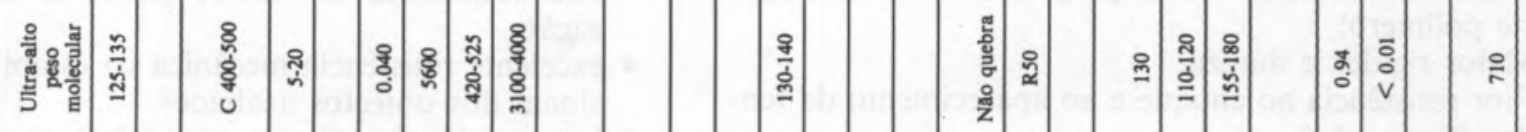

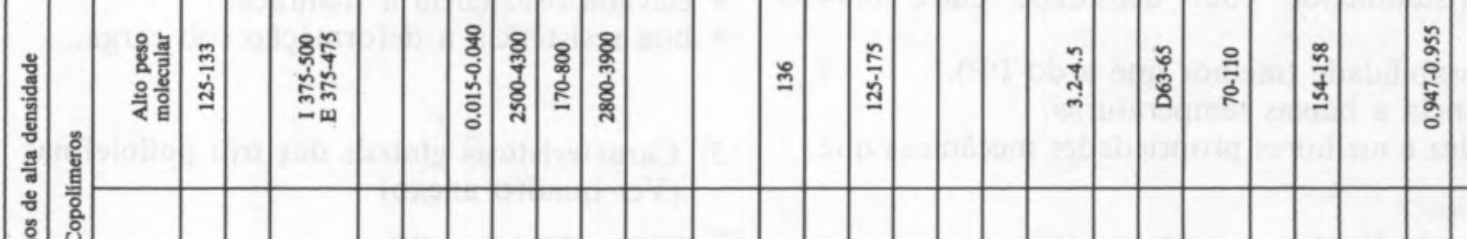

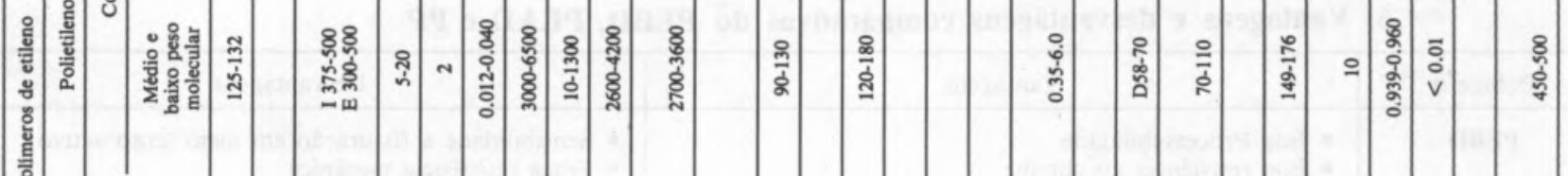
$\frac{2}{2}$

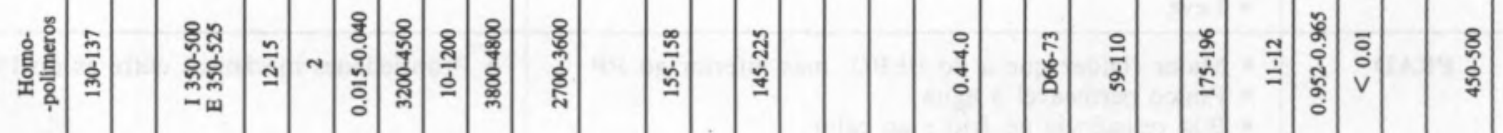



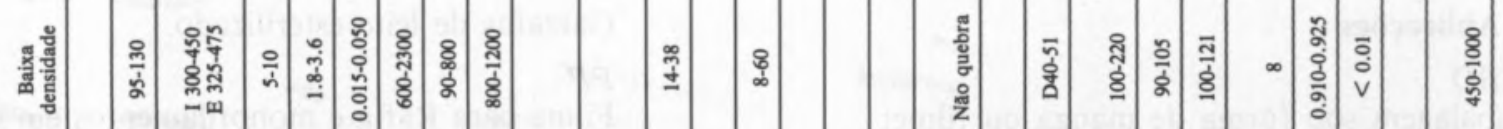

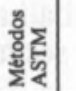



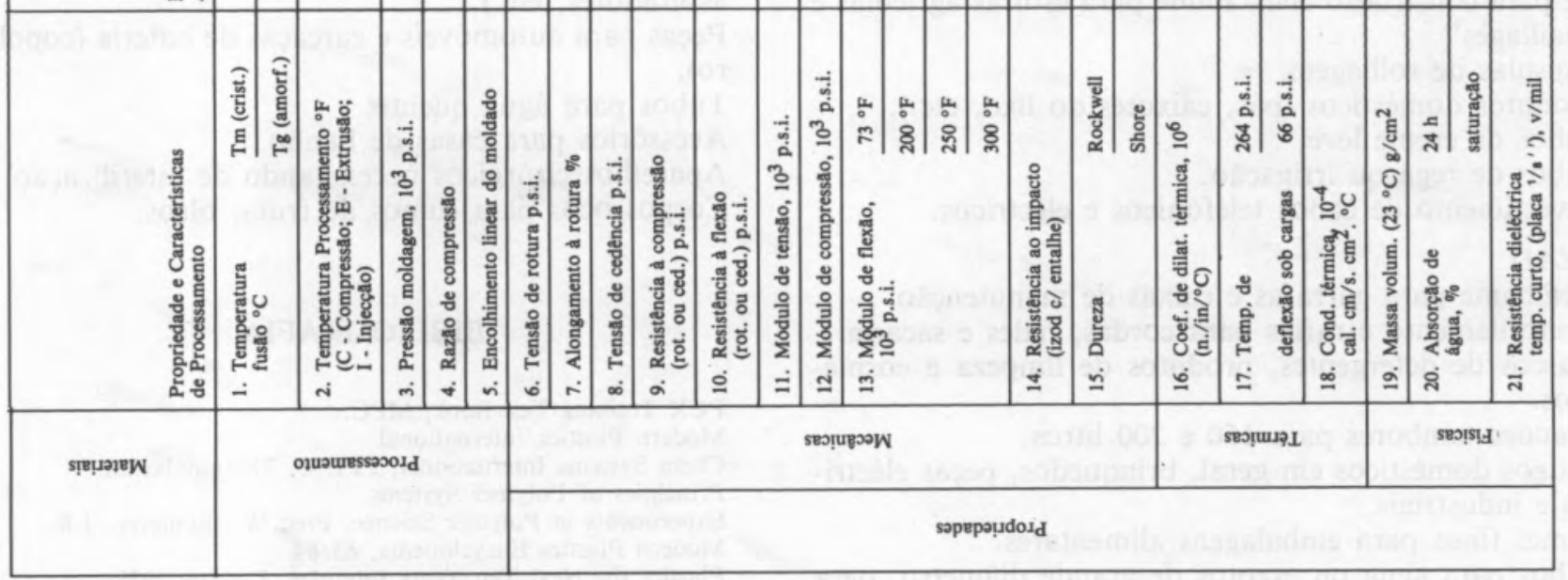

